\title{
Familial occurrence of congenital laryngeal clefts
}

\author{
P. D. PHELAN, J. G. STOCKS, H. E. WILLIAMS, and D. M. DANKS \\ From the Clinical Research Unit and Genetics Research Unit, Royal Children's Hospital Research Foundation; \\ Department of Anaesthesia, Royal Children's Hospital; and Department of Genetics, University of Melbourne, Melbourne, \\ Australia
}

Phelan, P. D., Stocks, J. G., Williams, H. E., and Danks, D. M. (1973). Archives of Disease in Childhood, 48, 275. Familial occurrence of congenital laryngeal clefts. Two sibships of double first cousins in which 6 children had congenital laryngeal anomalies are reported. 3 of the children had proven congenital posterior laryngeal clefts, 1 had subglottic stenosis with a deformed cricoid cartilage, and it is likely that the other 2 also had posterior laryngeal clefts. It is suggested that the anomaly is inherited as an autosomal dominant.

Congenital posterior cleft of the larynx is a rare anomaly, and fewer than 30 cases have been reported (Imbrie and Doyle, 1969). The extent of the cleft is variable; it may involve the larynx alone or extend to the trachea as far as the carina, resulting in an oesophagotrachea.

The occurrence of a laryngeal cleft in sibs has been reported twice previously. In one of the families, 2 children had posterior clefts and another 2 had stridor (Finlay, 1949; Crooks, 1954). In 1 the stridor was thought to be due to stenosis of the superior part of the larynx, in the other to an aberrant right subclavian artery. The other report was of two sibs with laryngeal clefts (Zachary

Received 14 August 1972. and Emery, 1961). The present report is 'of two sibships of double first cousins containing 6 children with congenital laryngeal anomalies, 3 of whom had proven laryngeal clefts.

\section{Clinical history}

The family tree is shown diagrammatically in the Fig. The fathers of the children were brothers (I.2, 4) and the mothers were sisters (I.14, 15). The 4 parents were well and none had a history of strider or of recurrent or persistent chest infections in infancy. The other members of the family were healthy and free of symptoms referable to laryngeal abnormalities, with the following exceptions. I.6 to 10 were 4 sisters and 1 brother of the mother who died in infancy of unknown causes.
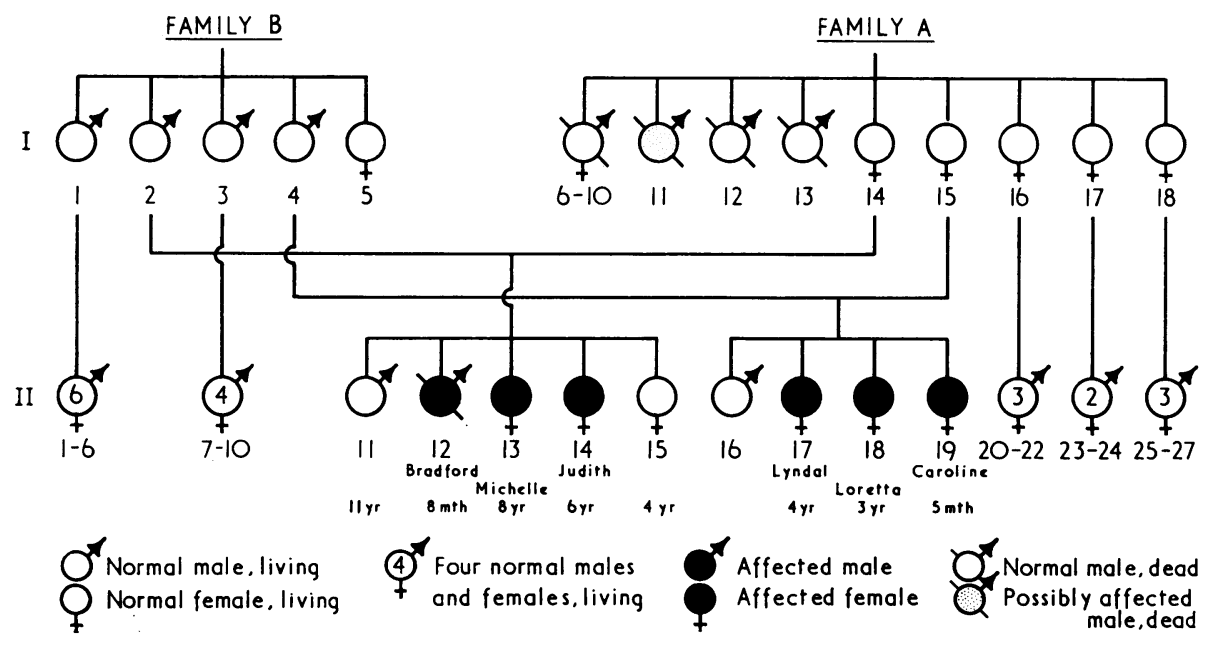

FIG.-Family tree showing the affected children. 
I.11 was a brother of the mother who was mentally retarded, had stridor all his life, and died at 31 years. I.12 and 13 were 2 brothers of the mother who died in adult life without symptoms suggestive of laryngeal disease.

\section{Affected children.}

Bradford (II.12). This child had stridor from soon after birth. There was no history suggestive of aspiration of milk into the respiratory passages but his stridor did become worse when he fed. He was admitted to the Royal Children's Hospital at the age of 5 months with severe laryngeal obstruction associated with respiratory infection, and a tracheostomy was performed. Considerable difficulty was experienced in his management, and it was never possible to extubate the baby. The largest endotracheal tube that could be passed through his larynx had an external diameter of 13 French gauge (approx. $4.3 \mathrm{~mm}$ ) and was a very tight fit. The child was found dead in his cot at the age of 10 months, probably as a result of mechanical obstruction of the tracheostomy tube.

At necropsy a subglottic stenosis was shown, the lumen in the subglottic region being $1 \times 4 \mathrm{~mm}$ after fixation in formalin. Serial sections of the larynx showed the cricoid to be mis-shapen and deficient posteriorly. No comment was made concerning the extent of the interarytenoid cleft.

Michelle (II.13). This child was transferred to the Royal Children's Hospital on the first day of life because of stridor and respiratory difficulties. An endotracheal tube of external diameter 14 French gauge (approx. $4.7 \mathrm{~mm}$ ) could be passed through her larynx with ease. She was a poor feeder, and physical and radiological signs of pulmonary infection persisted in her chest. During infancy and early childhood she would frequently cough when she drank fluids. A formal endoscopical examination of the child's larynx and trachea was never undertaken.

At the age of 8 she still had intermittent stridor and would cough if she drank fluids quickly. Her voice was soft but otherwise normal. Her chest was clinically and radiologically normal. Signs of precocious puberty were present.

Judith (II.14). This child coughed and spluttered when drinking fluids from the day she was born. She never had stridor. She was first seen at the age of 20 months because of a persistent cough. A chest $x$-ray at that stage showed irregular mottled opacities throughout the left lower lobe. Contrast medium spilled almost instantly through the larynx into the trachea when the child drank barium from a cup while in the sitting position.

No abnormality was detected in the larynx or tracheobronchial tree by endoscopy under general anaesthesia, but a laryngeal cleft was not specifically excluded.

She made reasonably satisfactory progress. At the age of 6 she was well but still coughed if she drank quickly. Her chest was clinically clear, but there were residual radiological changes in the lingula.
Lyndal (II.17). This child was admitted to the Royal Children's Hospital at the age of 4 days because she became cyanotic and coughed during feeding. At rest her respirations were quiet and there were no abnormal physical signs detectable. Swallowed barium was aspirated through the larynx into the trachea and major bronchi. Direct endoscopical examination of the larynx under general anaesthesia showed a posterior cleft extending at least to the level of the vocal cords. There was no subglottic narrowing.

Considerable difficulty was experienced in feeding this baby by mouth and a gastrostomy was performed at the age of 2 months. This was closed at the age of 5 months. From that time onwards the baby was able to cope with thickened feeds. She did have a number of episodes of bronchitis which were probably related to aspiration.

At the age of $4 \frac{1}{2}$ years she was well developed and had no abnormal physical signs in her chest. A chest $x$-ray was clear. Her mother commented that if she drank fluids quickly she would cough.

Loretta (II.18). This baby was fed with thickened feeds from birth because of the family history. She coughed and spluttered if she drank them quickly. A posterior laryngeal cleft was shown by direct laryngoscopy under general anaesthesia. The subglottic area was normal.

This baby has made satisfactory progress. At the age of 3 her chest was normal clinically and radiologically.

Caroline (II.19). This baby was noted to have an inspiratory stridor from birth. She was fed with thickened feeds but if these were inexpertly given she coughed and spluttered on them. Swallowed barium was aspirated into the trachea.

A posterior laryngeal cleft extending at least to the level of the vocal cords was shown endoscopically. She also had a subglottic stenosis with the trachea being narrowed anteriorly and laterally. A $2.5 \mathrm{~mm}$ internal diameter (approx. $4.0 \mathrm{~mm}$ external diameter) rubber endotracheal tube would not pass easily through the subglottic region when the baby was aged 7 weeks and weighed $5 \mathrm{~kg}$.

\section{Discussion}

Laryngeal clefts were certainly present in Lyndal, Loretta, and Caroline. On the basis of the history of coughing during feeding, it is highly likely that Michelle and Judith also had laryngeal clefts. Michelle was never examined endoscopically, and at the time of the endoscopical examination of Judith the authors were not aware of this anomaly. Bradford and Caroline had subglottic stenosis and Michelle had stridor, the cause of which was not determined.

The occurrence of laryngeal clefts in the 3 girls in the second sibship, and of proven subglottic stenosis in 1 boy in the first sibship, suggests that 
these lesions represent variants of one genetic phenotype. It seems very likely that the 2 girls with aspiration (Michelle and Judith) in the first sibship also had variants of the phenotype. None of the 4 parents had any relevant symptoms; however, examination under anaesthesia would be necessary to exclude a mild lesion, and this did not seem justified.

It would be quite possible for even a rare autosomal recessive phenotype to have been present in both groups of sibs since they were double first cousins. Alternatively, autosomal dominant inheritance would explain the observed distribution in the family if it assumed that either both mothers or both fathers carried the mutant gene without clinical symptoms. The fact that 5 sibs of the mothers died in infancy, and a sixth had stridor all his life, may suggest the presence of the gene in their generation. Even if this last information is ignored and if the 2 children with aspiration in the first sibship are regarded as affected, then one can calculate that autosomal dominant inheritance is statistically more probable than autosomal recessive, provided that at least 1 in 5 carriers of the mutant gene has no clinical manifestations. The variability of the symptoms in the affected children in this family makes this a reasonable assumption. Congenital laryngeal webs have also been reported as occasionally occurring as an autosomal dominant (Baker and Savetsky, 1966).

The published reports suggest that the majority of cases of laryngeal cleft are sporadic. However, there are two reports of multiple affected children born to symptom-free parents who do not appear to have been examined under anaesthesia. In the family reported by Zachary and Emery (1961) 2 of 4 sibs had laryngcal clefts and the other 2 were apparently normal. The family reported by Finlay (1949) and Crooks (1954) probably had other laryngeal anomalies associated with the laryngeal clefts. In 1 of the 2 children with stridor but without a laryngeal cleft, stenosis of the upper part of the larynx was shown at necropsy. In the other the stridor was attributed to an aberrant right subclavian artery, but this anomaly does not cause significant airways obstruction. One examiner thought the larynx was generally small, and it is probable that there was some unrecognized intrinsic laryngeal anomaly.

The association of subglottic stenosis and laryngeal cleft would suggest that the anomaly probably is a result of dysplasia of the cricoid cartilage. The exact nature of the subglottic obstruction was not determined at necropsy in Bradford and so it is impossible to say whether a cartilage bar arose from the cricoid; this is one of the common causes of congenital subglottic stenosis. Previous authors (Jahrsdoerfer, Kirchner, and Thaler, 1967) have suggested that laryngeal clefts result from failure of posterior fusion of the two wings of the cricoid cartilage, which arise in the sixth branchial arch. This failure of fusion may be associated with an arrest in the rostral advancement of the tracheo-oesophageal septum. Further disturbance in the development of the cricoid cartilage and associated tissues probably results in the subglottic stenosis.

The children reported here had the typical symptoms of a laryngeal cleft, which are coughing and difficulty with feeds. In Michelle and Judith the cause of this was not diagnosed because of lack of awareness of the condition. With thickened feeds the children were able to cope with their problem. They gradually developed trick movements in swallowing so that by middle childhood they were having little difficulty. Both mothers did comment that aspiration would still occur if their children drank quickly. Surgical repair of laryngeal clefts has been reported (Imbrie and Doyle, 1969), but the mortality and morbidity associated with the operation, especially in patients with long clefts, has been high. Obviously if the cleft is relatively small, as in the patients reported here, surgical repair is not necessary. These children do not seem to have any evidence of permanent lung damage.

This condition is probably not as rare as the few published case reports suggest. In addition to the family reported here, the authors have seen 4 other children with laryngeal clefts causing aspiration. One of these was associated with oesophageal atresia and tracheo-oesophageal fistula. It is probable that a number of cases are missed because the anomaly is not suspected or, if suspected, is not looked for appropriately. Simple inspection of the larynx is not adequate as the margins of the cleft can appear like a ruphe. The posterior wall of the larynx must be tested for a cleft by use of forceps which can separate the two sides. An endotracheal tube will also do this and lie posteriorly to the arytenoid cartilages. It is important that this particular cause of aspiration be identified so that correct treatment can be instituted.

Full resolution of the mode of inheritance will have to await further reports of families in which apparently unaffected parents happen to have been examined adequately. It is, however, difficult to justify laryngoscopy under general anaesthesia in a symptom-frce person. 


\section{REFERENCES}

Baker, D. C., and Savetsky, L. (1966). Congenital partial atresia of the larynx. Laryngoscope, 76, 616.

Crooks, J. (1954). Non-inflammatory laryngeal stridor in infants. Archives of Disease in Childhood, 29, 12.

Finlay, H. V. L. (1949). Familial congenital stridor. Archives of Disease in Childhood, 24, 219.

Imbrie, J. D., and Doyle, P. J. (1969). Laryngotracheosophageal cleft. Report of a case and review of the literature. Laryngoscope, 79, 1252.
Jahrsdoerfer, R. A., Kirchner, J. A., and Thaler, S. U. (1967). Cleft larynx. Archives of Otolaryngology, 86, 82 .

Zachary, R. B., and Emery, J. L. (1961). Failure of separation of larynx and trachea from the oesophagus; persistent oesophagotrachea. Surgery, 49, 525.

Correspondence to Dr. P. D. Phelan, Royal Children's Hospital, Flemington Road, Parkville, Victoria 3052, Australia.

A prize essay competition will be held, open to medical practitioners registered in the United Kingdom and qualified for not more than ten years. The value of the prize will be thirty pounds.

The subject of the essay shall be related to paediatrics but will be chosen individually by candidates. The essay shall consist substantially of unpublished work.

Entry forms and further information may be obtained from the Honorary Secretary, British Paediatric Association, c/o Institute of Child Health, 30 Guilford Street, London WC1N 1EH. The closing date for entries will be 30 June 1973 . 